must be satisfied with the axioms of connection and of parallels. In the upper grades, however, the student should learn what a rigorous and connected logical system geometry really is even if he does not carry the system through in detail. The speaker went on further to explain how and why the elements of projective geometry and nomography should be introduced. He closed with a short résumé of the actual state of instruction in Germany, and cited in behalf of his opinions the Arithmetik of Stolz and Gmeiner, the Encyclopedia of Elementary Mathematics by Weber and Wellstein, and the geometric treatises of Pasch, Hilbert, Veronese, and Ingrami.

19. Brückner, after some introductory remarks on the historical development of the problem of determining the equiangular, equisuperficial polyhedra - a problem solved for convex polyhedra by E. Hess - proceeded to give a résumé of the results he had found concerning the non-convex and discontinuous polyhedra of this type. Beside the sphenoids of the octahedral and icosahedral systems ( 7 and 5 groups, respectively) he went into the stephanoids of these two systems (3 and 11 combinations, respectively). The models of these discontinuous " null-polyhedra," and those of the continuous non-convex polyhedra with positive content, and those of Möbius's polyhedra of the icosahedral system were exhibited. (The complete results will be published in the Nova Acta of the Leopold Academy.)

Edwin BidWELl Wilson.

YALE UNIVERSITY.

\title{
THE BRESLAU MEETING OF THE DEUTSCHE MATHEMATIKER-VEREINIGUNG.
}

The annual meeting of the Deutsche Mathematiker-Vereinigung, forming a part, as usual, of the annual meeting of German scientists and physicians, was held this year at Breslau, in Silesia.

The first forenoon, Monday, September 19, was devoted to a general meeting of the entire association. In the afternoon of the same day the sessions of the different sections began. The convention consisted of 13 scientific and 17 medical sections, 
and not less than 650 papers and demonstrations were presented during the week.

In the mathematical section the attendance was very light, and but few papers were offered. It was evident that the Heidelberg congress, a few weeks previous, had practically cleared the field.

Although pure mathematics were somewhat neglected, this was offset by an unusual activity along pedagogical lines. On Thursday forenoon a general meeting was devoted to the topic: "Scientific and mathematical instruction in the higher secondary schools." The presence of representatives of the government and of teachers' associations gave evidence of a strong public interest in this subject. Professor A. Fricke (Bremen) opened the discussion with a paper on : "The present condition of instruction in natural science and mathematics in the schools of higher learning." He emphasized the fact that the German gymnasium is too much inclined along medieval lines; that is, the philological-historical side receives too much attention, while scientific instruction is not modified in accordance with new developments.

Professor Felix Klein then spoke on "New tendencies in the mathematical-physical field." He outlined the steps that had been taken to improve instruction in mathematics and physics, and then took up the methods used at present in teaching mathematics. He attributed the general aversion to mathematics in the gymnasia, not to the subject matter, but to the manner of presentation, and suggested as a remedy vacation courses in pedagogy for teachers, and a system whereby a teacher may obtain regular leave of absence in order to study and keep abreast of the times. Because of the position mathematics has assumed within the last century, as the basis for the exact sciences, he pleaded for an increase in the number of hours of instruction in mathematics at the expense of the ancient languages.

Other papers, bearing on instruction in biology and on school hygiene, were presented. A very animated general discussion followed with the result that a commission of twelve was appointed to consider the matter, and to report at the next meeting.

While the days were devoted to earnest work, the evenings were given up to social enjoyment. Monday evening occurred a presentation of the "Flying Dutchman" in the Stadt-theater; Tuesday evening, a banquet; Wednesday, small gatherings at 
different points; Thursday, supper in the Zoological garden followed by a boat ride on the Oder; and Friday evening, a banquet, fireworks, etc., after which the convention closed with a hearty "Auf Wiedersehen."

The following is a partial list of papers presented before the Mathematical section :

(1) Lampe (Berlin-Charlottenburg): "Some examples of the exercises in the integral calculus at the Charlottenburg technical school."

(2) Gutzmer (Jena): "The theory of linear homogeneous differential equations."

(3) Kowalewski (Greifswald): "On a generalization of the second mean value theorem of the integral calculus."

(4) Sturm (Breslau): "On the Cremona transformations in which to the planes of the one space correspond general surfaces of the third order in the other space."

(5) Landsberg (Heidelberg): "On the analogies between the theories of algebraic numbers and algebraic functions."

(6) Sternitz (Charlottenburg): "Collinear depictions of trigonal polyhedrons, and the analysis situs in projective space."

(7) LUdwig (Karlsruhe) : "On the theory of contact transformations of the circles on a sphere."

(8) Gutzmer (Jena): "On the theory of adjoined differential equations."

A brief abstract of each paper, so far as obtainable, follows :

1. In this paper is described the scope and character of the work in the Charlottenburg technical school during the first academic year, as it has been organized by Professor Lampe. As typical exercises, the following may be taken :

(a) Quadrature of the curves $(x / a)^{p}+(y / b)^{q}=1$ for $x>0$, $y>0$ from $x=0$ to $x=\alpha$ and for given values of $p$ and $q$, correct to a specified number of decimal places.

(b) Determination of the volumes inclosed by certain curved surfaces whose equations are first to be found ; e. g., given in polar coördinates $r, \phi$ the equation of the curve $r^{2}=a^{2} \cos ^{2} \phi$ $+b^{2} \sin ^{2} \phi$, a variable parabola has its vertex at a fixed point of the perpendicular to the plane of the curve through its centre; this perpendicular is the principal axis, and the parabola passes through the extremities of a diameter of the curve. The volume between the surface generated by the parabola and the given curve is to be found. Generalization of this exercise to other curves. 
(c) The conic $r=\rho /(1+e \cos \varphi)$ is given. On each radius vector $r$, as diameter, a circle is described in a plane perpendicular to the plane of the conic. Find the volume of the surface so generated in the several cases $e<1, e=1, e>1$.

2. Professor Gutzmer's first paper discussed the derivation of a method for determining the reciprocal differential equation and its employment in certain special cases in which it is particularly useful.

3-4. The papers of Professor Kowalewski and Professor Sturm will appear in the Jahresbericht.

5. Professor Landsberg compared the theory of algebraic numbers and of algebraic functions and called attention to certain analogies which these subjects furnish in their present state of development. His paper will appear in full in the Jahresbericht.

6. This paper treats of the depiction of a polyhedron whose faces are triangles upon a second polyhedron, whose faces are triangles, in such a way that a one-to-one correspondence exists between the vertices and between the faces of the two polyhedrons. Let $a, b, c, \cdots$ be the vertices of the first polyhedron and $a^{\prime}, b^{\prime}, c^{\prime} \ldots$ the corresponding vertices of the second. In order to depict one triangle $a b c$ on the corresponding triangle $a^{\prime} b^{\prime} c^{\prime}$, it is only necessary to associate an arbitrary point in the first with an arbitrary point in the second. If we continue this process we come finally to a point on an edge $a b$ which belongs to two triangles and can be represented on $a^{\prime} b^{\prime}$ in two ways. At this point the one-to-one correspondence fails, but it can be maintained if the projective representation of the edges is fixed. It is necessary in order to bring two edges in relation to select a point pair $p, p^{\prime}$. This is analytically possible if the value of $a^{\prime} p^{\prime} \mid p^{\prime} b^{\prime}: a p / p b$ or the real logarithm is given. The last value changes sign with a change in direction. The selection of the projective relation is, then, not arbitrary. Geometrically the condition is, that the lines joining the midpoints of the sides of the triangle $a b c$ to the vertices $a^{\prime} b^{\prime} c^{\prime}$ must pass through one point. Analytically, the condition is, that the sum of the logarithms belonging to any three sides of a triangle must be zero. We will call the logarithm belonging to any edge $(a b)$ its integral value and denote it by $\int_{a}^{b}$. From the fore- 
going we have then $(a b)=-(b a),(a b)+(b c)+(c a)=0$; as in the theory of abelian integrals of the first and second kinds, we conclude here that every complete integral whose path is a simply connected surface must vanish. One obtains here a group of $\infty^{e-1}$ solutions ( $e$ denotes the number of vertices) when one gives arbitrary functional values $f(\alpha), f(b), \cdots$ to each of the vertices $a, b, c \cdots$ and defines the integral values of the edges $a b, \cdots$ by means of the equation

$$
\int_{a}^{b}=f(b)-f(a)
$$

This last result does not change when the functional values of the vertices are increased by a constant quantity. In this manner only those solutions are exhausted whose complete integral is zero. The presence of moduli of periodicity in the case of integrals of the first and second kinds shows that still other solutions exist. In fact, in the case of a bilateral polyhedron there are in all $\infty^{(e-1)+z}$ solutions, where $z=k+2-$ $e-f=2 p . \quad k=$ number of edges, $e=$ number of vertices, $f=$ number of surfaces, $p=$ genus.

In the case of a unilateral polyhedron there are $\infty^{(e-1)+(z-1)}$ solutions. A close connection exists between this problem and certain investigations in the topology of projective space, which gives the following result. While in euclidean space corresponding points on two surfaces which in the sense of analysis situs are equal, $i$. e., permit of a one-to-one depiction on each other, can be made to coincide, in projective space this is not the case. Each type of analysis situs, from this point of view, falls into $2^{z}$ subtypes.

7. A purely geometric investigation of the contact transformations of the circle on a sphere requires that one consider them as applied to the entire sphere and in their complete multiplicity. It is therefore necessary to start from very meager conditions, $i$. $e$, a contact transformation $L$ of the circle must associate with every point on the sphere a circle and transform every circle into two circles (which may coincide). The same must be true for the inverse transformation $L^{-1}$. It appears that this transformation contains ten constants. Lie had shown by a different method that all contact transformations of a circle on a sphere form a ten-element group. The author shows that these two methods lead to the same group $L$. 
8. The second paper by Professor Gutzmer consists of a short report on some investigations and results related to a previous paper by him under the title: "Theory of adjoined differential equations," Halle, 1896.

The next meeting of the Vereinigung will be held at Meran, in the Tyrol, in September, 1905, under the presidency of Professor F. Klein.

Göttingen, Germany,

R. E. WIILSON.

November, 1904.

\section{THE CONSTRUCTION OF CONICS UNDER GIVEN CONDITIONS.}

BY PROFESSOR M. W. HASKELL.

(Read before the San Francisco Section of the American Mathematical Society, April 30, 1904.)

UNDER the above title are grouped a few notes on special aspects of the subject, with no pretense at an exhaustive treatment. While certain of the results are not new, it is believed that the method by which they are derived will be of some interest. Homogeneous coördinates are used exclusively ; following Casey (Analytical Geometry, page 70), a quadrangle or quadrilateral whose diagonal triangle is the triangle of reference is designated as a "standard" quadrangle or quadrilateral. The coördinates of the vertices of a standard quadrangle, or of the sides of a standard quadrilateral, are then $\pm \kappa_{1}: \pm \kappa_{2}: \pm \kappa_{3}$, and they may be projected into $\pm 1: \pm 1: \pm 1$ if desired. It is to be noticed that the complete quadrangle (quadrilateral) is fully determined and can be easily constructed, when the diagonal triangle and any one vertex (side) are given.

§1. Conics Defined by Five Points or by Five Tangents.

Theorem I. The eight vertices of two standard quadrangles lie on one and the same conic.

For, let the vertices be respectively $\pm \kappa_{1}: \pm \kappa_{2}: \pm \kappa_{3}$ and $\pm \lambda_{1}: \pm \lambda_{2}: \pm \lambda_{3}$; it is clear that they all lie on the conic whose equation is 